Received: 13 March 2019

Accepted: 10 July 2019

Published online: 22 July 2019
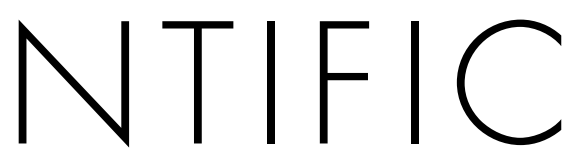
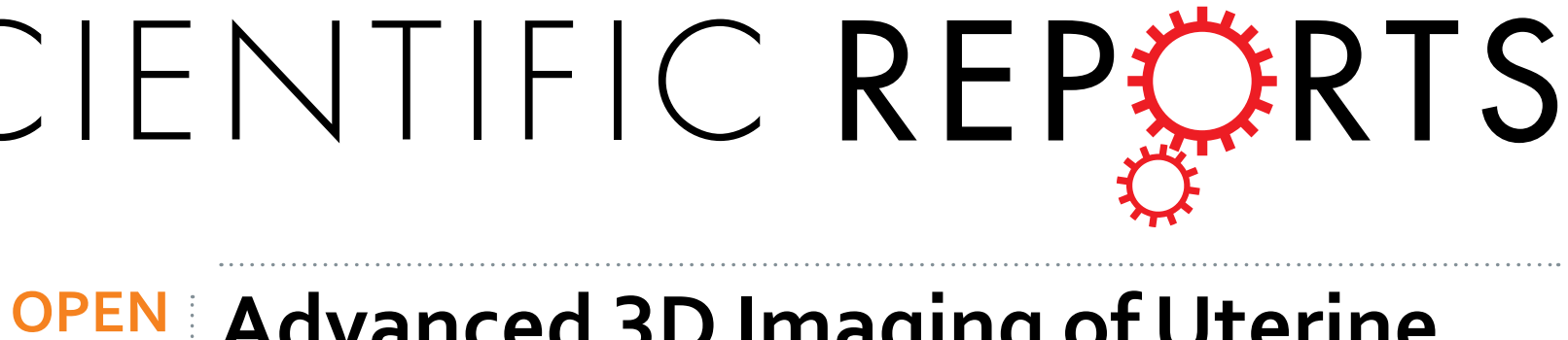

\title{
Advanced 3D Imaging of Uterine Leiomyoma's Morphology by Propagation-based Phase-Contrast Microtomography
}

\author{
Alessandra Giuliani ${ }^{1}{ }^{1}$, Stefania Greco², Serena Pacilè ${ }^{3}$, Alessandro Zannotti ${ }^{1,2}$, \\ Giovanni Delli Carpini ${ }^{1}$, Giuliana Tromba ${ }^{3}$, Stefano Raffaele Giannubilo ${ }^{1}$, Andrea Ciavattini ${ }^{1}$ \& \\ Pasquapina Ciarmela
}

Uterine leiomyoma is the most common benign smooth muscle tumor in women pelvis, originating from the myometrium. It is caused by a disorder of fibrosis, with a large production and disruption of extracellular matrix (ECM). Medical treatments are still very limited and no preventative therapies have been developed. We supposed that synchrotron-based phase-contrast microtomography (PhCmicroCT) may be an appropriate tool to assess the 3D morphology of uterine leiomyoma, without the use of any contrast agent. We used this technique to perform the imaging and the quantitative morphometric analysis of healthy myometrium and pathologic leiomyomas. The quantitative morphometric analysis of collagen bundles was coupled to the Roschger approach. This method, previously only used to evaluate mineralized bone density distribution, was applied here to study the fibrosis mass density distribution in healthy and pathologic biopsies from two patients. This protocol was shown to be powerful in studying uterine leiomyomas, detecting also small signs of the ECM alteration. This is of paramount importance not only for the follow-up of the present study, i.e. the investigation of different compounds and their possible therapeutic benefits, but also because it offers new methodologic possibilities for future studies of the ECM in soft tissues of different body districts.

Uterine leiomyoma (myoma or fibroid) is the most common benign smooth muscle tumor in women pelvis causing significant morbidity in a large segment of reproductive-aged women ${ }^{1,2}$, including significant impact on the reproductive healthy status and on the pregnancy outcome of affected patients $s^{3,4}$.

Fibroids remain the leading indication for hysterectomy $y^{5,6}$ especially during the peri-menopause period, although the assumption that leiomyoma symptoms will resolve with the onset of the menopause is too simplistic and may not be always valid ${ }^{7}$. Medical treatments for leiomyoma are still very limited and no preventative therapies have been developed ${ }^{8}$.

The precise pathogenesis of uterine leiomyoma is not well understood. Genetic alterations, epigenetic mechanisms, steroids, growth factors, cytokines and chemokines provide the clue of initiators and promoters of leiomyoma growth ${ }^{9}$. Uterine myoma is thought to be a consequence of myofibroblasts activation and extracellular matrix (ECM) production, following an improper inflammatory response inside the myometrium ${ }^{10-12}$. Excessive accumulation of ECM components including collagens, fibronectin and proteoglycans are the major structural part of leiomyoma tissue that are abnormally orientated modifying mechanical stress on cells and leading to activation of internal mechanical signaling and dynamic reciprocity ${ }^{13-15}$. The ECM stiffness causes the rigid structure of the leiomyoma and it is thought to be a cause of abnormal bleeding and pelvic pressure and pain ${ }^{16}$.

The role of inflammation and reparative processes as well as the effect of some potential therapeutic compounds (tranilast, strawberry extracts, omega-3 fatty acids, genistein, natural fitoterapeutics, vitamin D) on uterine leiomyoma are currently under in vitro investigation ${ }^{17-29}$.

${ }^{1}$ Department of Clinical Sciences, Università Politecnica delle Marche, Ancona, Italy. ${ }^{2}$ Department of Experimental and Clinical Medicine, Università Politecnica delle Marche, Ancona, Italy. ${ }^{3}$ Elettra Sincrotrone Trieste S.C.p.A, Trieste, Italy. Correspondence and requests for materials should be addressed to A.G. (email: a.giuliani@univpm.it) or P.C. (email: p.ciarmela@univpm.it) 
At the moment, structural laboratory studies of myometrial and fibrotic tissue are limited to the two-dimensional (2D) morphological investigation. Only an advanced three-dimensional (3D) characterization of the fibrotic tissue structure can enhance our knowledge of the tissue architecture, significantly supporting the development of an effective therapeutic solution. For example, the in-vivo efficiency of therapeutic compounds could be hampered, even if delivered locally, by their inability to reach the leiomyoma cells due to the fibrotic hard and perhaps not permeable tissue architecture. Therefore, it would be essential to evaluate the whole 3D tissue structure.

Nowadays, X-ray tomography (CT) is not the method of choice for the characterization of pelvic masses; indeed, uterine fibroids are detected incidentally by CT scans, often performed for other reasons. Medical ultrasonography (USG) is usually the initial investigation tool to examine the female pelvis, performing both transabdominal and transvaginal scans. Magnetic resonance imaging (MRI) is the favourite method for an accurate characterization of pelvic masses. Indeed, MRI was shown to be more sensitive in identifying uterine fibroids than USG, and it has the advantage, respect to CT, that it does not involve the use of ionizing radiation ${ }^{30,31}$.

However, up to now, literature has not definitely clarified if the discrimination between healthy and pathologic myometrium is just a matter of stiffness or there are also any density variations. For instance, the typical CT images show fibroids that may appear morphologically complex, with hypodense, though they may be isointense, and rarely hyper intense areas. Calcification is seen in approximately $4 \%$ of fibroids: it is typically dense and amorphous, sometimes confined to the periphery of the fibroids. As such, the CT appearance of leiomyomas cannot reliably be distinguished from uterine or cervical neoplasms $\mathrm{s}^{32}$.

$\mathrm{X}$-ray phase-contrast imaging (XRPCI) techniques may constitute a potential powerful tool for non-invasive myometrium imaging, in healthy and pathologic conditions and without the use of any contrast agent. Indeed, differently from the X-ray absorption-based imaging, where the contrast originates from attenuation mismatches between different tissues inside a sample, in the XRPCI the contrast is due to the phase-shift $\delta$ of the refractive index $n=1-\delta+i \beta$, describing the interaction of the X-ray beam throughout the material ${ }^{33,34}$. This $\delta$ value, in non-mineralized biological tissues like the myometrium, can be up to three orders of magnitude larger than attenuation values $\beta^{35,36}$, achieving an highly increased contrast, as observed investigating several organs/tissues, including brain ${ }^{37,38}$, vessels ${ }^{39-41}$, kidney ${ }^{42}$, neuronal system ${ }^{43}$, cartilage ${ }^{44,45}$ and breast tissues ${ }^{46,47}$. Moreover, ten years ago, synchrotron radiation-based high resolution phase-contrast tomography (SR-PhC-microCT) was also used, in a pioneering way, for the study of the ECM organization within polymeric scaffolds ${ }^{48}$.

No XRPCI studies, to the proponents' best knowledge, have been performed so far on the uterine myometrium and leiomyomas without the use of a contrast agent. Thus, in the present demonstrative study, we aimed to test the use of the propagation-based phase-contrast imaging to discriminate healthy and pathologic uterine tissues.

\section{Results}

Taking into account that the slight nominal mismatch between collagen $\left(1.41 \mathrm{~g} / \mathrm{cm}^{3}\right)^{49}$ and muscle $\left(1.055 \div 1.112 \mathrm{~g} / \mathrm{cm}^{3}\right)^{50}$ mass densities would have prevented, most likely, a reliable analysis by absorption-based imaging, all the 15 biopsies were investigated by PhC-microCT and subsequently reconstructed exploiting the Paganin's method ${ }^{51}$ for the phase retrieval processing.

Representative 2D slices of healthy myometrium (Ctr) and leiomyoma (L) tissues are shown in Fig. 1 (panels a-d) for both patients P1 and P2. Stack-sequences of 2D axial slices (subvolumes, each with a final volume of $600 \times$ $600 \times 400 \mu^{3}$ ) have been reported for representative samples of P1 in Movies 1 and 2 in the Supplementary Material section. 3D reconstructions of the same volumes are shown in Fig. 1 (panels e,f), where all the tissues but the collagen phase have been made virtually transparent. Morphological dissimilarities were clearly found between Ctr and L samples of the same patient (P-Ctr vs. P-L) and between pathologic tissues of the two patients (P1-L vs. P2-L). The analysis was also able to reveal differences between the healthy tissues of the two patients (P1-Ctr vs. P2-Ctr).

In agreement with previous literature, mainly based on histologic findings, the pathologic biopsies revealed an higher amount of collagen than the healthy myometrium. Moreover, distribution and orientation of the collagen bundles were very varied in the different samples, suggesting the need for a detailed morphometric analysis.

The quantitative analysis was performed with an approach based on two levels of investigation.

In the first level, the application of the Mixture Modeling algorithm ${ }^{52}$ to the histograms (based on an unsigned 8-bit scale of grey) of the full set of samples indicated a threshold of 110 between muscle and collagen grey levels, achieving the collagen morphometric quantification, as detailed in Table 1 and illustrated in Fig. 2.

The specific surface (CollS/CollV) and the mean distance between the collagen bundles (Sp) in the leiomyoma were lower than in the healthy myometrium. Coherently, the specific volume (CollV/TV), the mean thickness (Th) and the mean number $(\mathrm{Nr})$ of collagen bundles were higher than in the healthy myometrium.

Unexpectedly, three other results were obtained, as detailed in Table 1:

(1) although the number of samples analyzed was comparable, the standard deviation of data obtained for P1Ctr was always higher than for data related to P2-Ctr;

(2) the Nr was higher in P1-Ctr than in P2-Ctr;

(3) the Connectivity Density (Conn.D) was comparable between P1-Ctr and P1-L but lower in P2-Ctr than in P2-L.

In general, these three results, revealed large structural inhomogeneity inside myometrial and leiomyoma tissues.

Moreover, up to now, literature has not definitely clarified if the discrimination between healthy and pathologic myometrium is just a matter of stiffness or there are also density variations. Consequently, we achieved the 

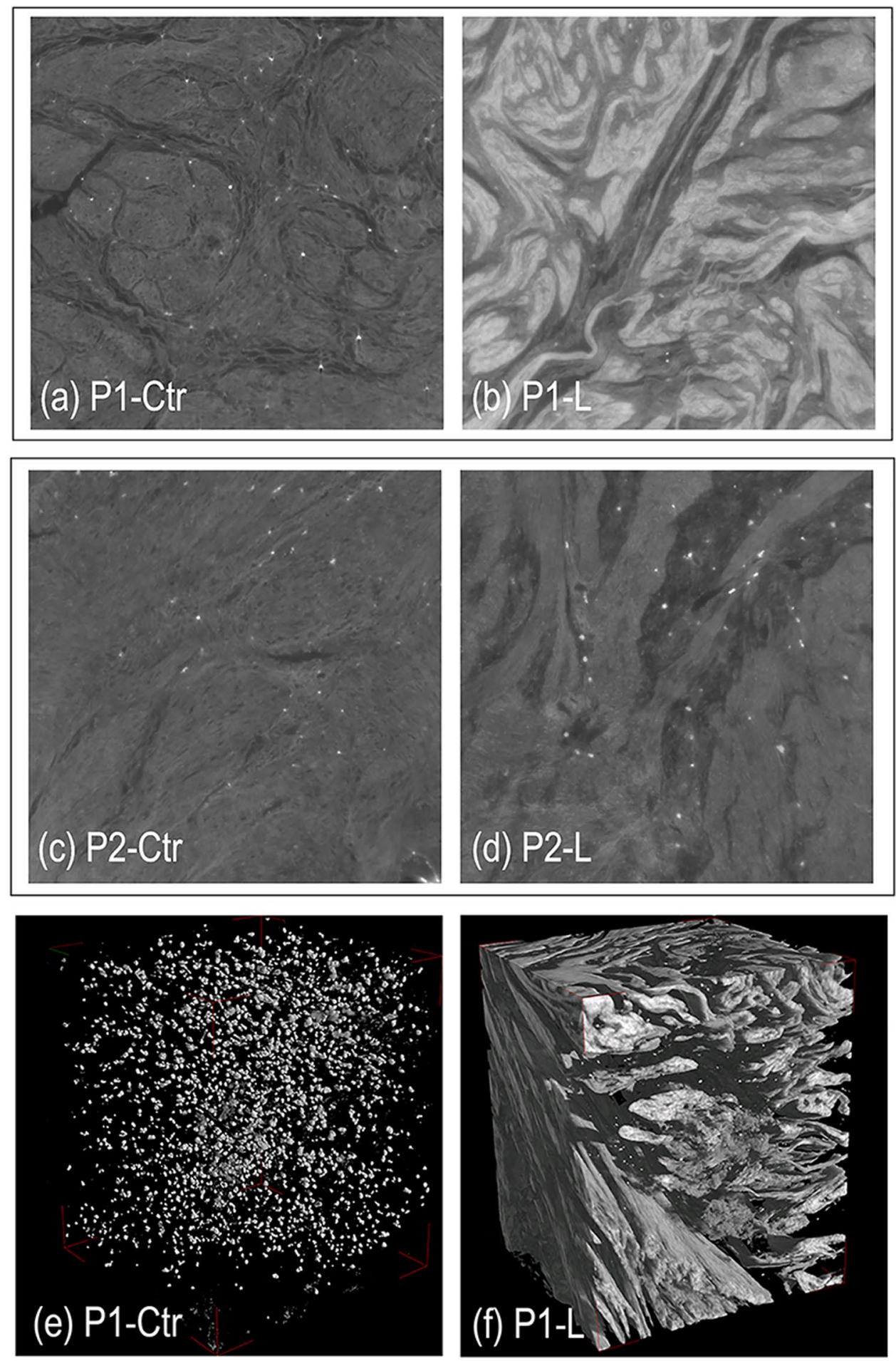

Figure 1. (a-d) Representative 2D slices of healthy myometrium (Ctr) and pathologic (L) tissues. (a,b) Patient 1 (P1): healthy myometrium (a: P1-Ctr) and leiomyoma (b: P1-L) tissues; (c,d) Patient 2 (P2): healthy myometrium (c: P2-Ctr) and leiomyoma (d: P2-L) tissues. Morphological dissimilarities were found not only between Ctr and L samples of the same patient and between pathologic tissues of the two patients (P1-L vs. P2-L) but also between the healthy tissues of the two patients (P1-Ctr vs. P2-Ctr). (e,f) Representative 3D volumes of healthy myometrium (e: P1-Ctr) and pathologic (f: P1-L) tissues: all the tissues but the collagen phase have been made virtually transparent. The bright spots that we found in all the samples were due, most likely, to the presence of blood clots residues, not removed by washing because of the structure tortuosity.

second level of the quantitative analysis. It was based on the approach designed by Roschger ${ }^{53}$ and, to date, it was succesfully used to evaluate the mineral density distribution in bone (BMDD) ${ }^{54,55}$. Indeed, the Roschger method delivered fundamental descriptors of the mineral density distribution of the bone matrix throughout a sample: 


\begin{tabular}{|l|l|l|l|l|}
\hline & P1-Ctr & P1-L & P2-Ctr & P2-L \\
\hline CollS/CollV $\left[\mathrm{mm}^{-1}\right]$ & $388 \pm 213$ & $63 \pm 20$ & $422 \pm 117$ & $200 \pm 71$ \\
\hline CollV/TV $[\%]$ & $15.0 \pm 24.4$ & $70.5 \pm 10.9$ & $3.1 \pm 2.1$ & $32.6 \pm 15.8$ \\
\hline $\mathrm{Th}[\mu \mathrm{m}]$ & $7.8 \pm 6.8$ & $35.4 \pm 13.1$ & $5.0 \pm 1.2$ & $11.4 \pm 4.9$ \\
\hline $\mathrm{Nr}\left[\mathrm{mm}^{-1}\right]$ & $13.0 \pm 10.7$ & $21.1 \pm 3.8$ & $6.0 \pm 3.2$ & $28.7 \pm 4.4$ \\
\hline Sp $[\mu \mathrm{m}]$ & $145.9 \pm 125.2$ & $13.9 \pm 3.7$ & $211.3 \pm 126.4$ & $23.9 \pm 7.1$ \\
\hline DA & $0.378 \pm 0.081$ & $0.632 \pm 0.089$ & $0.399 \pm 0.166$ & $0.378 \pm 0.081$ \\
\hline Conn.D $\left[\times 10^{-7}\right.$ pixel $\left.^{-3}\right]$ & $234.6 \pm 368.5$ & $259.4 \pm 52.5$ & $45.0 \pm 58.3$ & $741.9 \pm 472.2$ \\
\hline
\end{tabular}

Table 1. Collagen three-dimensional morphometric analysis in the retrieved biopsies. Mean values \pm standard deviation. P1-Ctr: heathy myometrium in patient 1; P1-L: leiomyoma in patient 1; P2-Ctr: healthy myometrium in patient 2; P2-L: leiomyoma in patient 2.

thus, it was shown to play a fundamental role in testing if either diseases and/or treatments might be of significant biological and clinical relevance ${ }^{53}$.

Thus, starting from the rational that the leiomyoma pathology was shown to be connected to the excessive accumulation of collagen, the study of fibrosis mass density distribution by the Roschger's approach was supposed to help in clarifying if distribution deviations from healthy conditions were involved. We tested the Roschger method descriptors (Fig. 3a) to evaluate, for the first time to the authors' knowledge, the apparent uterine mass density distribution $\left(\mathrm{MDD}^{\mathrm{r}}\right)$. Similarly to the previous studies, absolute values of mass density could not be retrieved because they might have been biased by the used constant delta-over-beta ratio in the Paganin phase retrieval ${ }^{56}$. However, being the different biopsies comparable in terms of size and composition, the quantitative comparison of the relative difference in mass density distribution between the samples is feasible. Thus, hereinafter the superscript $r$ will denote relative values for all mass density distribution parameters.

The quantitative data obtained in the investigated biopsies were reported in the box plots of the Fig. 3b-f. Median values of the relative mass density distribution in P1-Ctr were found lower than in P1-L; on the contrary, the fwhm and the high (99.5th percentile) values were higher in the leiomyoma in comparison to the healthy controls for both the patients. However, as expected after the morphometric analysis of collagen, the fwhm and the high values of P1-Ctr were higher than those obtained in P2-Ctr. Therefore, it must be concluded that some or all the biopsies derived from patient 1 , clinically considered non-pathological tissue portions, were actually composed by very heterogenic samples, in terms of stiffness and density distribution.

\section{Discussion}

The role of the extracellular matrix (ECM) in the biomechanics of the human uterus has just been begun to be studied in recent years. The ECM does not only surround cells, but its rigidity stresses them mechanically, producing signals that depend on the amount of collagen, its cross-linking and hydration. Therefore, the ECM can induce the so-called mechano-transduction process, namely cells convert a mechanical stimulus into an electrochemical activity. Indeed, the etiology of uterine fibroids indicates that its growth is due to an increase not only of the cell number but also of the ECM amount, both promoted by endocrine and autocrine growth factors. In turns, the alterations in ECM volume and distribution can modify mechanical stress on cells, leading to activation of specific cell's signaling that contribute to leiomyoma growth ${ }^{16,57}$. Therefore, alterations of morphology and/or composition of the ECM can be important signals of the onset and of the evolution of this pathology.

Previous studies showed an impaired expression of collagen, fibronectin and proteoglycans in leiomyoma compared to normal myometrium ${ }^{58-61}$ : in particular, leiomyoma was shown to contain an abnormal collagen fibril structure and orientation, suggesting that the well-regulated fibril formation in myometrium is altered in leiomyomas. In fact, an important study showed that the interaction of two or more different types of fibrillar collagen chains may interact and result in the formation of heterotypic fibrils, missing in leiomyomas, that in myometrium would assist in regular fibril formation in normal uterine tissue. In this context, alterations in collagen may play a role in the pathogenesis of leiomyomas ${ }^{62}$. Thus, the study of specific collagen parameters, such as the size of collagen bundles, orientation and interconnectivity, turns out to be particularly interesting because its morphometry should determine specific cellular behaviors. This is similar to what happens in bone sites, although the interpretation of what morphometric measurements mean for collagen masses in the myometrium must be related to the specific myometrial tissue and its function.

Therefore, we have focused our analysis on the study of collagen. The propagation-based phase-contrast microCT was used, for the first time to the authors' knowledge, to evaluate the collagen amount and distribution in intramural fibroids excised from the uterus of two fertile women.

In our study, we observed higher volumes, thickness and number of collagen bundles in the leiomyoma compared to normal adjacent myometrium. In the case of patient 1, the interconnectivity of the collagen structure was also increased in the pathological tissue compared to the control. These data obtained on collagen networks justify the increase in the rigidity of the pathological tissue compared to the healthy one and they are in agreement with the observation performed with conventional 2D techniques, like histology and electron microscopy, in several studies found in literature.

The specific surface (CollS/CollV), defined as the surface area per unit volume, is an architectural parameter that can contribute to determine type and properties of a material. This measure is strongly dependent from shape: if volume is held constant, matrix of different shapes will typically adopt different specific surface values. Interestingly, we found that collagen specific surface in leiomyoma is lower than in normal counterpart. Certainly, 
a

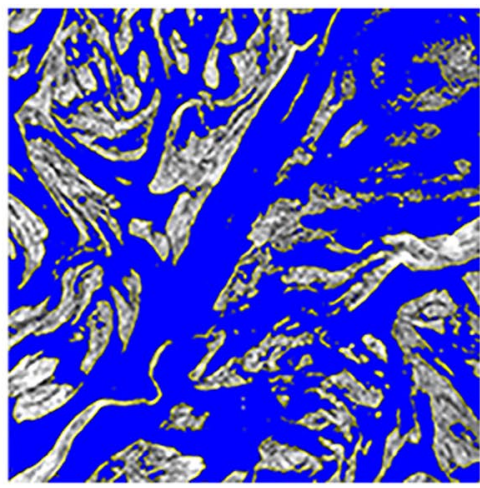

C

Specific Surface (CollS/CollV)
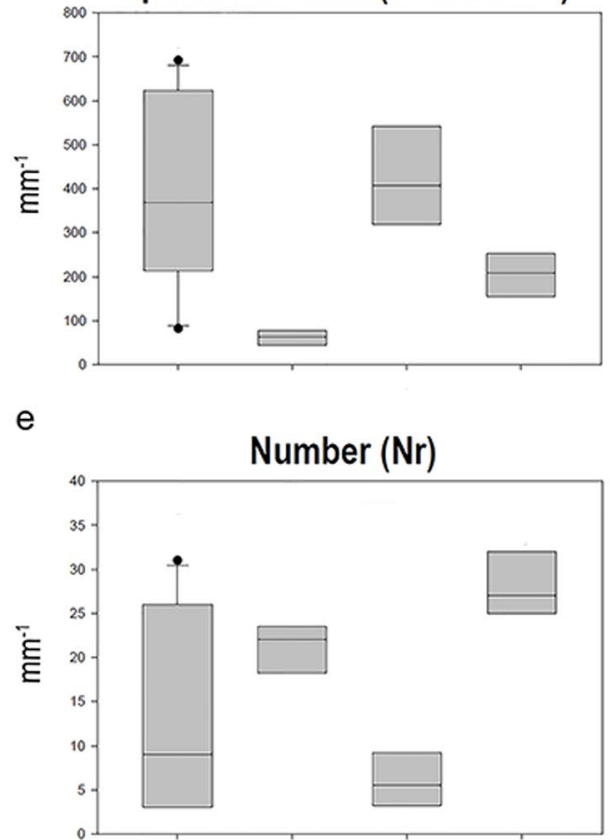

g

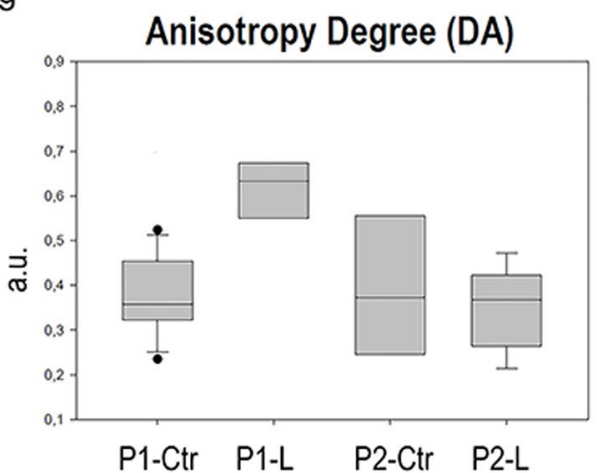

b

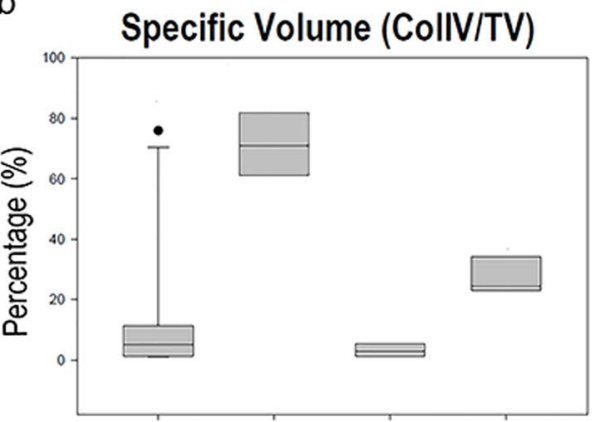

d

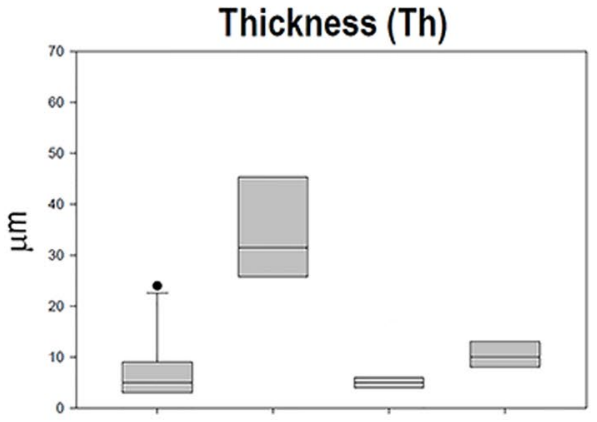

f

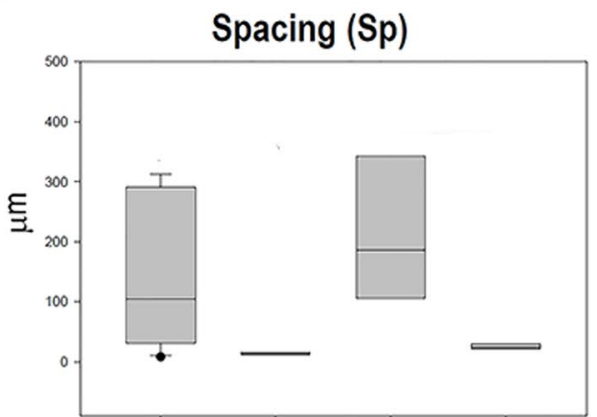

h

\section{Connectivity Density (Conn.D)}

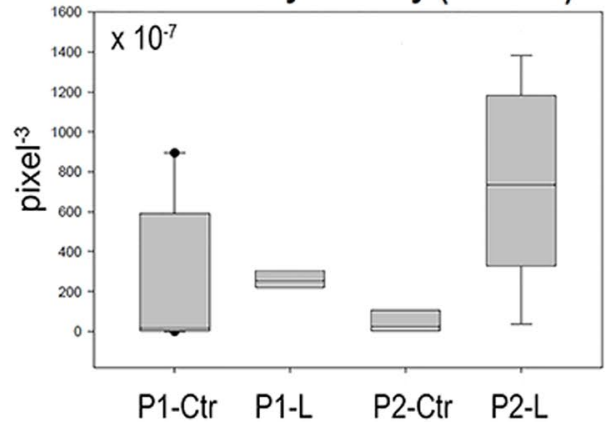

Figure 2. 1st level analysis: study of the collagen morphometric quantification. (a) Sampling 2D slice of a pathologic biopsy where the histogram has been segmented according to the application of the Mixture Modeling algorithm. Blue phase: smooth muscle; graded grey phase: collagen bundles. (b-h) Box-plots graphically depicting groups of the extracted collagen morphometric parameters through their quartiles, as a function of the selected groups of samples P1-Ctr, P1-L, P2-Ctr and P2-L.

this is due to the fragmentation of collagen in myometrium versus the aggregation of collagen in leiomyoma. To the authors' knowledge, collagen specific surface has never been measured in myometrium or leiomyoma tissue, although it could represent an important index to study, due to relevant clinical implications. The presence of focal nodal collagen distribution in the myometrium, as in the case of uterine fibroids, could alter the normal 
a

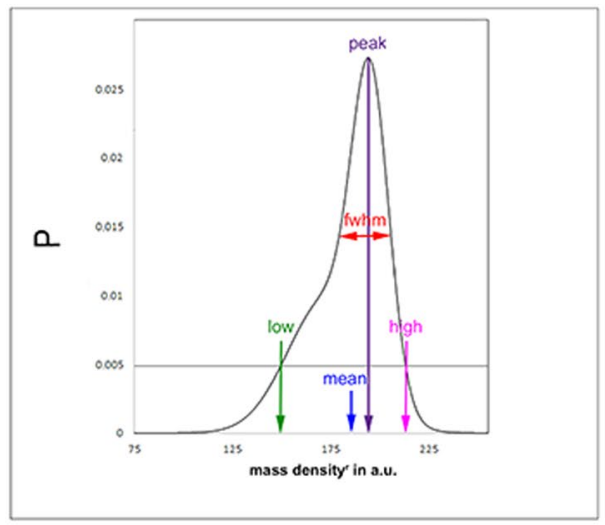

C

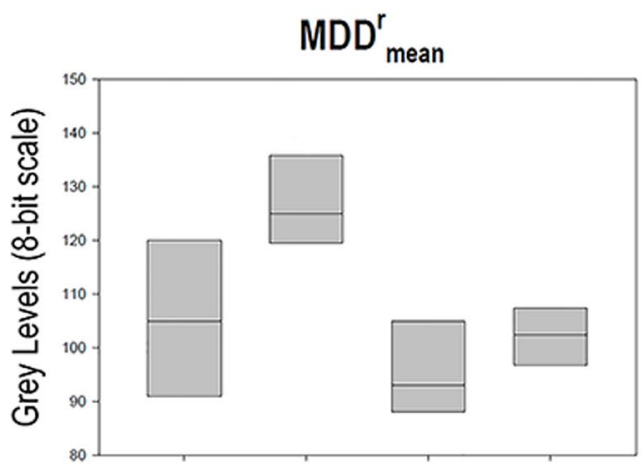

e

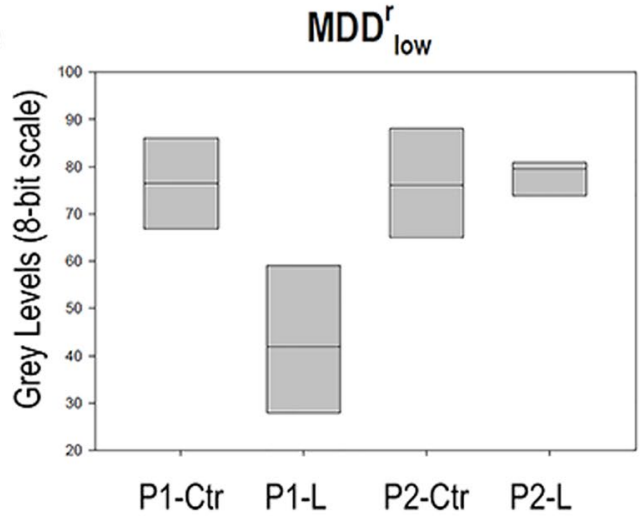

b

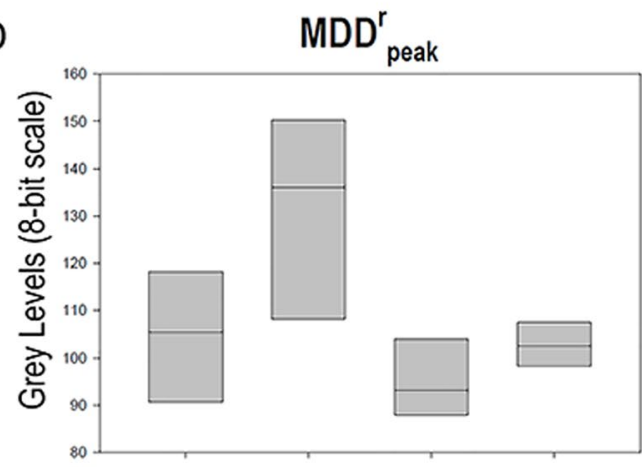

d

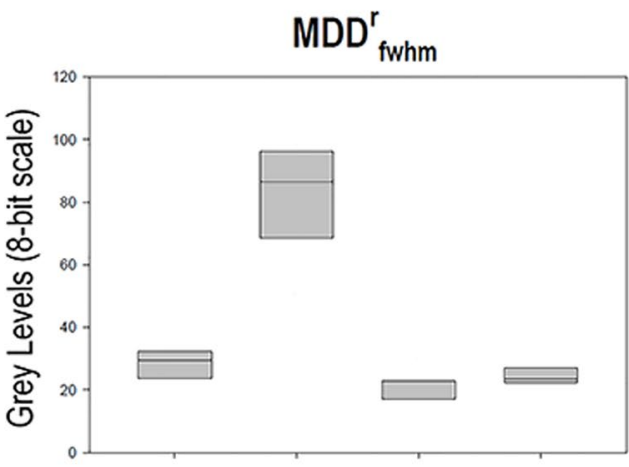

f

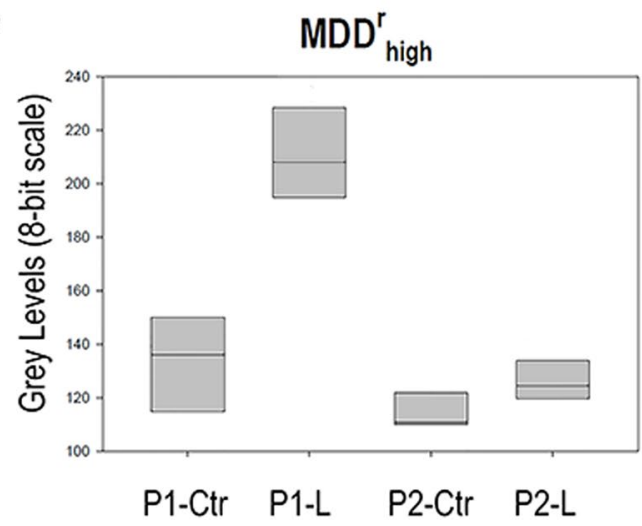

Figure 3. 2nd level analysis: study of the Relative Mass Density Distribution $\left(\mathrm{MDD}^{\mathrm{r}}\right)$. (a) The parameters derived from the profile fitting are indicated. (b-f) Box-plots graphically depicting the extracted $\mathrm{MDD}^{\mathrm{r}}$ parameters through their quartiles as a function of the selected groups of samples P1-Ctr, P1-L, P2-Ctr and P2-L.

uterine peristalsis through mechanical interference. Indeed, it was reported that patients with intramural fibroids may present abnormal uterine peristalsis during the mid-luteal phase. Since uterine peristalsis is involved in normal reproductive processes, this condition seems to reduce the possibility of obtaining spontaneous pregnancy ${ }^{63}$. Moreover, the study from Yoshino and colleagues highlighted how myomectomy reduced the frequency of abnormal peristalsis in all patients ${ }^{64}$. Collagen specific surface may become a useful parameter to perform further studies, i.e. comparing normal myometrial tissues versus fibroid tissues, as well as to study different type of fibroids (i.e. large vs small or pre-menopausal vs post-menopausal or benign vs malignant).

Although the present study should be considered prevalently as a set-up of a new study methodology, we consider appropriate to point out that the structural properties of fibroids and even of healthy myometrium tissues differ widely within the same tissue and among the different patients.

We and others have recently observed that the amount of collagen inside tissues within and between biopsies is heterogeneous mainly in leiomyoma, but also in myometrial tissue. For example, morphometric analysis of Masson's trichrome stained tissue reported values of collagen in leiomyoma ranging from $4.7 \%$ to $31,3 \%$ in our previous study ${ }^{11}$; from $37 \%$ to $77 \%$ in Jayes ${ }^{65}$ study and from $3.9 \%$ up to $70 \%$ in a large study of brazilian cases $(\mathrm{n}=150)$ performed by Da Silva ${ }^{66}$. Flake and colleagues categorized uterine leiomyoma in 4 different phases on the basis of percent collagen content ranging from none (phase 1) up to $72 \%(>50 \%$, phase 4 ) independently of 
the tumor size ${ }^{67}$. The collagen content in myometrium ranged from $0.3 \%$ to $23 \%$ in our previous samples ${ }^{11}$ and from $1.6 \%$ up to $20.70 \%$ in Da Silva's study ${ }^{66}$. Interestingly, the leiomyoma tissues appeared inhomogeneous also in stiffness, quantified through rheometry by measuring complex shear moduli of the tissues. Fibroid tissues, with stiffness of $8014 \pm 798 \mathrm{~Pa}$ (mean \pm SEM), were stiffer than myometrial samples $(3630 \pm 276 \mathrm{~Pa})^{65}$. With the same indication, evidence of biomechanical and biochemical heterogeneity in uterine fibroids has just been reported. The authors observed heterogeneity in structure, collagen content, and stiffness highlighting that fibroid regions differ in biochemical status and they suggest that these differences might be associated with variations in local pressure, biomechanical signaling, and altered growth ${ }^{68}$.

All this opens new prospective to understand uterine fibroids and to explain how it is possible that, despite of the same hormonal milieu, different fibroids of the same subject have a different fate and degree of growth. These differences in fibroids structure could also explain the heterogeneous effect of fibroids of the same dimensions, number, and localization on fertility, on response to medical therapy, and on pregnancy outcomes.

Therefore, the present and all these recent studies, provide novel evidences that structural properties of uterine leiomyoma and, even if less pronounced, also of the myometrium are widely heterogeneous. The variability within and among tissues should be considered in order to characterize the samples, to design and conduct studies to understand the pathobiology as well as to test potential treatments.

Moreover, it has to be stressed that physical, topological, and biochemical composition of the ECM is not only tissue-specific, but it is also markedly heterogeneous: ECM is a highly dynamic structure that is constantly being remodeled, as it happens in bone sites. For instance, collagen density distributions in human knee ligaments were documented to quantify differences in density within and between these ligaments ${ }^{69}$. Very recently, heterogeneity in structure, collagen content, and stiffness in uterine fibroids was reported ${ }^{68}$. Thus, it is interesting to evaluate if this heterogeneity is expressed also in terms of density distributions, as it was shown in human knee ligaments.

In our study, we performed the analysis of fibroid mass density distribution by the Roschger's approach. We found that the full width at half maximum and the 99.5th percentile values were significantly higher in the leiomyoma in comparison to the healthy adjacent controls for both the patients. This means that, in pathological biopsies, not only the amount of collagen is greater (as found out by the morphometric analysis) but also its density distribution is wider than in controls, with presence of highly fibrotic and high-density areas.

Another remark that deserves to be made concerns the density variability of healthy myometrial tissue.

Indeed, using the Roschger approach we showed that, comparing healthy myometrial tissues, the full width at half maximum and the 99.5th percentile values of patient 1 were higher than the respective ones obtained in patient 2, confirming that some tissue portions clinically classified as healthy, showed not only differences in ECM volume but also in density distribution.

On the other hand, it should not be surprising to realize that myometrium of fibromatous uterus is not a homogeneous tissue; it presents structural and functional alterations and consequently it cannot be considered completed healthy. For example, just consider that in the myometrium surrounding the leiomyoma there is accumulation of inflammatory cells ${ }^{11}$. Furthermore, irregular thickening of the endomyometrial junctional zone due to inordinate proliferation of the inner myometrium, junctional zone hyperplasia, is a common MR finding in women suffering from menstrual dysfunction ${ }^{70,71}$.

In this context, a textural analysis would have been additionally informative; however, its application in this demonstrative study would have been challenging and perhaps not completely reliable. Indeed, in order to have a reliable textural analysis, the pixel size should be decreased to at least $500-600 \mathrm{~nm}$, in order to fully resolve the texture also in the healthy myometrium (in Fig. 1e the spotty-signal reveals only the larger nodes of the collagen structure).

In conclusion, the propagation-based phase-contrast microCT was shown to be a powerful method in studying uterine leiomyomas, detecting also small signs of the ECM alteration.

In general, the PhC-microCT was shown to provide 3D images of intact tissues as well as a wide range of numerical indices that can be calculated and used to identify mismatches between different tissues. This is of fundamental importance with a view to follow up these studies in various directions: first of all, to visualize the 3D structure of the ECM in myometrium and pathologic tissues (different forms of leiomyoma, as well as leiomyosarcoma); secondly, to test ex-vivo in toto tissue the efficacy of innovative therapeutic treatments for leiomyomas; more generally, to offer new methodological possibilities for future studies on ECM in soft tissues of different body districts.

\section{Methods}

Sample collection and Permissions. Samples of myometrial tissue and leiomyoma were excised from two fertile women submitted to hysterectomy. The patients were Caucasians (age: 49 years and 43) and the position of the leiomyomas was intramural: the former with a single mass of $10 \mathrm{~cm}$, the latter with two masses of $4 \mathrm{~cm}$ and $2 \mathrm{~cm}$. Both patients displayed good general condition; none of them had a history of myomectomy or uterine surgery, had received medical therapy or oral contraceptives in the previous three months, or had evidence of genital tract infection, endometriosis, or ovarian disease. Patients gave their informed consent and the permission of the Human Investigation Committee was granted (Ethics Committee of Marche Region, Prot. N 2015 0486OR).

All experiments were performed in accordance with relevant guidelines and regulations.

The samples, immediately after hysterectomy, were collected in Hanks' Balanced Salt Solution (HBSS) (Euroclone, Milan, Italy) and transferred to the laboratory for washing them with Dulbecco's PBS (Invitrogen, Life Technologies, Carlsbad, CA, USA) in order to remove excess of blood and cutting them into small pieces.

In the former patient (P1), four pieces were taken from the fibrotic tissue (P1-L) and other four from the healthy myometrium (P1-Ctr); in the latter patient (P2), four pieces were taken from the leiomyoma (P2-L) and other three from the healthy myometrium (P2-Ctr). 
Synchrotron Radiation microCT examination. The SR-microCT experiments were performed at the SYRMEP beamline of the ELETTRA Synchrotron Facility (Basovizza, TS, Italy).

SR-microCT in its simplest form is based on the inversion of the Radon transform of the experimental projections. After the so-called flat fielding of the projection, the filtered back projection (FBP) algorithm is applied to the corrected data in order to reconstruct the stack of $2 \mathrm{D}$ slices ${ }^{72}$. The conventional $\mathrm{x}$-ray imaging approach is based on the discrimination of the different attenuation properties of the elements composing the imaged object, that are related to $\beta$, the complex part of the index of refraction $n=1-\delta+i \beta$. In this case, image contrast is generated by differences in $\mathrm{x}$-ray absorption. However, because of the coherence of SR, also the electron density, related to the phase shift term $\delta$, might be exploited leading to phase-contrast imaging ${ }^{33}$. With this approach, applicable only if X-ray wavefield has high coherence characteristics, the setup is sensitive to detect not only the absorption differences but also the phase shifts occurring to $\mathrm{x}$-rays crossing the sample. This approach becomes fundamental when the discrimination between two materials having similar electron densities or negligible X-ray absorption is required. Indeed, the reconstruction of $\delta$ distribution improves the image contrast, thus simplifies the image segmentation and the subsequent quantitative analysis.

We used this last method to investigate the 3D morphology of the fibrotic tissue and the mass density distribution in the biopsies retrieved from patients affected by leiomyoma.

The scans were performed using the pink SR beam provided by the machine, filtered by $0.5 \mathrm{~mm}$ Silicon and corresponding to an average energy of $19 \mathrm{keV}$; we used $0.2 \mathrm{~s}$ of exposure time per projection over a total range of $180^{\circ}$; the sample-detector distance was set to $100 \mathrm{~mm}$, resulting in $1 \mu \mathrm{m}^{3}$ isotropic voxel size in the reconstructed 3D images. The tomographic reconstruction was performed using the SYRMEP Tomo Project (STP) open source software ${ }^{72}$. Including the intensity of the recorded radiographs a phase contrast signal, a phase retrieval algorithm, exploiting the Paganin's method ${ }^{51}$, was applied to the (flat-corrected) projection data in order to reconstruct the decrement $\delta$ of the refractive index $n$. In the Paganin's method, the phase is retrieved by simply assuming a linear relationship between the absorption index $\beta$ and the refractive index decrement $\delta$. The approximation is valid for homogenous samples and propagation distances in the near field regime ${ }^{51}$, conditions fulfilled for the considered samples and the adopted scans geometries. The $\delta / \beta$ ratio was set to 100 .

Afterwards, the commercial software VG Studio MAX 1.2 (Volume Graphics, Heidelberg, Germany) was used to generate $3 \mathrm{D}$ images, where grey levels were proportional to the phase distribution. Optimal image quality was achieved by setting the Scatter HQ algorithm with an oversampling factor of 5.0.

Image analysis. The presence of different phases within the biopsies translated into different peaks in the gray-level scale. Thus, the volume of each phase was obtained by multiplying the volume of a voxel by the number of voxels underlying the peak associated with the relevant phase. The Mixture Modeling algorithm, implemented as plugin in the NIH ImageJ software (https://imagej.net/Mixture_Modeling_Thresholding and https://imagej. nih.gov/ij/plugins/mixture-modeling.html) ${ }^{73}$, was chosen to threshold the portion of the histograms related to the two adjacent main tissues, namely the smooth muscle and the fibrotic collagen-based phase. The Mixture Modeling algorithm is a histogram-based technique that assumes that the histogram distribution is represented by two Gaussian curves. It calculates the image threshold as the intersection of these two Gaussians, finding a threshold that is, in several cases, very close to real world data. In our histogram data, the Gaussian curve on the left was representative of the smooth muscle, the second curve on the right of the collagen-phase. Indeed, in this range of densities should be included also the endothelial signal but the thickness of vessels, if excluding the pregnancy period, is negligible, not affecting the segmentation process. Therefore, we analyzed in each pathological biopsy, with the aforementioned algorithm, a dozen slices, uniformly selected in the axial direction: the selected threshold value was the average of all those obtained. Notably, for the selection of this threshold value, control biopsies were not examined because they were considered unreliable due to their low collagen content. An example of histogram segmented by the Mixture Modeling algorithm has been reported for a representative sample of P1-L in Fig. 4 of the Supplementary Material section.

For each biopsy, several subvolumes were analyzed: each of them was a 3D portion fully included in the sample bulk and the complete set of them allowed to achieve the complete sample mapping.

The quantitative analysis was performed in two steps.

In the first step, the morphometric evaluation of the fibrotic collagen-based phase was made using the structural indices usually measured for bone samples ${ }^{74}$ : the collagen specific volume (CollV/TV, expressed as a percentage), its specific surface (CollS/CollV, per millimeter), the mean collagen bundles thickness (Th, expressed in micrometers), the mean collagen bundles number ( $\mathrm{Nr}$, per millimeter) and the mean collagen bundles spacing (Sp, expressed in micrometers). Furthermore, as collagen bundles could vary their orientation depending on the pathology, we also extracted information about the anisotropy of the collagen structure, i.e. the presence of preferential orientation(s). The anisotropy degree index (DA) measures the similarity of a fabric to a uniform distribution and varies between 0 , representing all observations confined to a single plane or axis, and 1 , corresponding to the perfect isotropy. The DA analysis was performed using the BoneJ Plugin ${ }^{75}$ of the ImageJ software ${ }^{73}$, version 3. The morphometric analysis was also extended to the inclusion of a descriptor for the interconnectivity of the collagen bundles: its connectivity density (Conn.D - pixel $^{-3}$ ) does not carry information about positions or size of connections, but it is a simple global measure of connectivity that gives higher values for better-connected structures, i.e. more entangled fibrotic tissues, and lower values for poorly connected ones, i.e. well-oriented bundles.

In the second step, the reconstructed complex refractive index distribution was exploited ${ }^{54}$; indeed, it is linearly related to the mass density and was assessed computing the relative mass density distribution $\left(\mathrm{MDD}^{\mathrm{r}}\right)$ of each sample, as shown in Fig. 3a. In each slice-image, the individual grey level voxels reflect the average density found in the corresponding volume elements of the investigated sample region: consequently, the acquisition of grey 
level voxel images provided the information on the local variation of density throughout the tissue. This variation in density is best described and quantified by a frequency distribution (histogram), as shown in the Fig. 3a. In order to compare different mass density distributions between biopsies, a reduction of the huge amount of histogram data to only few characteristic parameters is necessary. Briefly, the $\mathrm{MDD}^{\mathrm{r}}$ was calculated within the overall tissue domain and was normalized by the area under the curve.

The absolute values of mass density could not be retrieved because the reconstructed complex refractive index might be biased by the chosen $\delta / \beta$ ratio used in the Paganin phase retrieval processing ${ }^{56}$. Thus, being the different samples comparable in terms of size and composition, the superscript $r$ was used to denote relative values for all mass density distribution parameters. The Roschger approach ${ }^{53}$ was used, for the first time to the authors' knowledge, to study tissues different from bone.

Five parameters were extracted from the $\mathrm{MDD}^{\mathrm{r}}$ profile of each sample: $\mathrm{MDD}^{\mathrm{r}}$ peak , the peak position of the histogram, which indicates the most frequently measured density (density with the highest number of pixels); $\mathrm{MDD}_{\text {mean }}^{\mathrm{r}}$, the mean relative mass density obtained from the integrated area under the curve; $\mathrm{MDD}_{\text {fwhm }}^{\mathrm{r}}$, the full width at half maxima of the distribution, describing the variation in density; $\mathrm{MDD}_{\text {low }}^{\mathrm{r}}$ the percentage of the area that has a density below the 5 th percentile of the reference range; and the $\mathrm{MDD}^{\mathrm{r}}$ high , the percentage of the area that has a density above the $95^{\text {th }}$ percentile of the reference range. The threshold of $\mathrm{P}=0.005$ was arbitrarily chosen because it was considered a good compromise between maintaining good sensitivity for low and high values in the $\mathrm{MDD}^{\mathrm{r}}$ and minimizing potential artifacts due to partial volume effect in the evaluation of $\mathrm{MDD}_{\text {low. }}^{\mathrm{r}}$ This post-processing calculation of the $\mathrm{MDD}^{\mathrm{r}}$ parameters was performed using the PeakFit software (Systat Software, San Jose, CA).

\section{References}

1. Ciarmela, P. et al. Growth factors and myometrium: biological effects in uterine fibroid and possible clinical implications. Human Reproduction Update 17(6), 772-790 (2011).

2. Day Baird, D. et al. High cumulative incidence of uterine leiomyoma in black and white women: ultrasound evidence. American Journal of Obstetrics and Gynecology 188(1), 100-107 (2003).

3. Ciavattini, A. et al. Number and size of uterine fibroids and obstetric outcomes. J Matern Fetal Neonatal Med 28(4), 484-8 (2015).

4. Ciavattini, A. et al. Growth trend of small uterine fibroids and human chorionic gonadotropin serum levels in early pregnancy: an observational study. Fertil Steril 105(5), 1255-1260 (2016).

5. Cardozo, E. R. et al. The estimated annual cost of uterine leiomyomata in the United States. American journal of obstetrics and gynecology 206(3), 211. e1-211. e9 (2012).

6. Downes, E. et al. The burden of uterine fibroids in five European countries. European Journal of Obstetrics, Gynecology, and Reproductive Biology 152(1), 96-102 (2010).

7. Ciarmela, P. et al. Management of leiomyomas in perimenopausal women. Maturitas 78(3), 168-73. (2014).

8. Islam, M. S. et al. Uterine leiomyoma: available medical treatments and new possible therapeutic options. Journal of Clinical Endocrinology \& Metabolism 98(3), 921-934 (2013).

9. Islam, M. S. et al. Complex networks of multiple factors in the pathogenesis of uterine leiomyoma. Fertility and Sterility 100(1), 178-193 (2013).

10. Leppert, P., M. Fouany \& J. H. Segars Understanding uterine fibroids, in Fibroids, J.H. Segars, Editor. 2013, John Wiley \& Sons, Ltd: Oxford. p. 1-10

11. Protic, O. et al. Possible involvement of inflammatory/reparative processes in the development of uterine fibroids. Cell \& Tissue Research 364, 415-427 (2016).

12. Protic, O. et al. Activin A in Inflammation, Tissue Repair, and Fibrosisossible Role as Inflammatory and Fibrotic Mediator of Uterine Fibroid Development and Growth. Semin Reprod Med 35(6), 499-509 (2017).

13. Leppert, P. C., Jayes, F. L. \& Segars, J. H. The extracellular matrix contributes to mechanotransduction in uterine fibroids. Obstet Gynecol Int 2014, 783289 (2014).

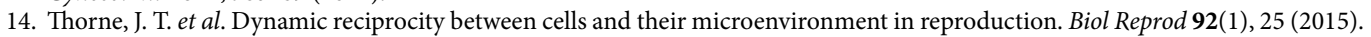

15. Rafique, S., Segars, J. H. \& Leppert, P. C. Mechanical Signaling and Extracellular Matrix in Uterine Fibroids. Semin Reprod Med 35(6), 487-493 (2017).

16. Islam, M. S. et al. Extracellular matrix in uterine leiomyoma pathogenesis: a potential target for future therapeutics. Hum Reprod Update 24(1), 59-85 (2018).

17. Islam, M. S. et al. Use of dietary phytochemicals to target inflammation, fibrosis, proliferation, and angiogenesis in uterine tissues: Promising options for prevention and treatment of uterine fibroids? Molecular nutrition \& food research 58(8), 1667-1684 (2014).

18. Islam, M. S. et al. Tranilast, an orally active antiallergic compound, inhibits extracellular matrix production in human uterine leiomyoma and myometrial cells. Fertility and sterility 102(2), 597-606 (2014).

19. Islam, M. S. et al. Antiproliferative effect of tranilast on human myometrial and leiomyoma cells. Biological and Biomedical Reports 2(5), 321-327 (2012).

20. Islam, M. S. et al. Dietary phytochemicals for possible preventive and therapeutic option of uterine fibroids: Signaling pathways as target. Pharmacological Reports 69(2017), 57-70 (2016).

21. Islam, M. S. et al. Molecular targets of dietary phytochemicals for possible prevention and therapy of uterine fi broids: Focus on fi brosis. Critical Reviews in Food Science and Nutrition 57(17), 3583-3600 (2017).

22. Islam, M. S. et al. An anthocyanin rich strawberry extract induces apoptosis and ROS while decreases glycolysis and fibrosis in human uterine leiomyoma cells. Oncotarget 8(14), 23575-23587 (2017).

23. Islam, M. S. et al. Omega-3 fatty acids modulate the lipid profile, membrane architecture, and gene expression of leiomyoma cells. $J$ Cell Physiol 233(9), 7143-7156 (2018).

24. Giampieri, F. et al. Romina: A powerful strawberry with in vitro efficacy against uterine leiomyoma cells. J Cell Physiol (2018).

25. Castro, L. et al. A High Concentration of Genistein Induces Cell Death in Human Uterine Leiomyoma Cells by Autophagy. Expert Opin Environ Biol, 5(Suppl 1) (2016).

26. Di, X. et al. A high concentration of genistein down-regulates activin A, Smad 3 and other TGF- $\beta$ pathway genes in human uterine leiomyoma cells. Experimental \& molecular medicine 44(4), 281-292 (2012).

27. Di, X. et al. A low concentration of genistein induces estrogen receptor-alpha and insulin-like growth factor-I receptor interactions and proliferation in uterine leiomyoma cells. Hum Reprod 23(8), 1873-83 (2008).

28. Moore, A. B. et al. Stimulatory and inhibitory effects of genistein on human uterine leiomyoma cell proliferation are influenced by the concentration. Hum Reprod 22(10), 2623-2631 (2007).

29. Ciavattini, A. et al. Hypovitaminosis D and "small burden" uterine fibroids: Opportunity for a vitamin D supplementation. Medicine (Baltimore) 95(52), e5698 (2016). 
30. Hossain, M. Z. et al. A Comparative Study of Magnetic Resonance Imaging and Transabdominal Ultrasonography for the Diagnosis and Evaluation of Uterine Fibroids. Mymensingh Med J 26(4), 821-827 (2017).

31. Levens, E. D. et al. Magnetic resonance imaging and transvaginal ultrasound for determining fibroid burden: implications for research and clinical care. Am J Obstet Gynecol 200(5), 537 e1-7 (2009).

32. Ascher, S. M. \& Silverman, P. M. Applications of computed tomography in gynecologic diseases. Urol Radiol 13(1), 16-28 (1991).

33. Bravin, A., Coan, P. \& Suortti, P. X-ray phase-contrast imaging: from pre-clinical applications towards clinics. Phys Med Biol 58(1), R1-35 (2013).

34. Snigirev, A. et al. On the possibilities of $\mathrm{x}$-ray phase contrast microimaging by coherent high-energy synchrotron radiation. Review of Scientific Instruments 66(12), 5486-5492 (1995).

35. Lewis, R. A. et al. X-ray refraction effects: application to the imaging of biological tissues. Br J Radiol 76(905), 301-8 (2003).

36. Momose, A. et al. Phase-contrast X-ray computed tomography for observing biological soft tissues. Nat Med 2(4), 473-5 (1996).

37. Croton, L. C. P. et al. In situ phase contrast X-ray brain CT. Sci Rep 8(1), 11412 (2018).

38. Pinzer, B. R. et al. Imaging brain amyloid deposition using grating-based differential phase contrast tomography. Neuroimage 61(4), 1336-46 (2012).

39. Giuliani, A. et al. Synchrotron Phase Tomography: An Emerging Imaging Method for Microvessel Detection in Engineered Bone of Craniofacial Districts. Front Physiol 8, 769 (2017).

40. Cedola, A. et al. X-Ray Phase Contrast Tomography Reveals Early Vascular Alterations and Neuronal Loss in a Multiple Sclerosis Model. Sci Rep 7(1), 5890 (2017).

41. Vagberg, W. et al. Cellular-resolution 3D virtual histology of human coronary arteries using x-ray phase tomography. Sci Rep 8(1), 11014 (2018).

42. Velroyen, A. et al. X-ray phase-contrast tomography of renal ischemia-reperfusion damage. PLoS One 9(10), e109562 (2014).

43. Bukreeva, I. et al. Quantitative 3D investigation of Neuronal network in mouse spinal cord model. Sci Rep 7, 41054 (2017).

44. Coan, P. et al. Characterization of osteoarthritic and normal human patella cartilage by computed tomography X-ray phase-contrast imaging: a feasibility study. Invest Radiol 45(7), 437-44 (2010).

45. Horng, A. et al. Cartilage and soft tissue imaging using X-rays: propagation-based phase-contrast computed tomography of the human knee in comparison with clinical imaging techniques and histology. Invest Radiol 49(9), 627-34. (2014).

46. Grandl, S. et al. Visualizing typical features of breast fibroadenomas using phase-contrast CT: an ex-vivo study. PLoS One 9(5), e97101 (2014).

47. Pacile, S. et al. Clinical application of low-dose phase contrast breast CT: methods for the optimization of the reconstruction workflow. Biomed Opt Express 6(8), 3099-112 (2015).

48. Albertini, G. et al. Organization of extracellular matrix fibers within polyglycolic acid-polylactic acid scaffolds analyzed using X-ray synchrotron-radiation phase-contrast micro computed tomography. Tissue Eng Part C Methods 15(3), 403-11 (2009).

49. Noda, H. Partial specific volume of collagen. J Biochem 71(4), 699-703 (1972).

50. Ward, S. R. \& Lieber, R. L. Density and hydration of fresh and fixed human skeletal muscle. J Biomech 38(11), 2317-20 (2005).

51. Paganin, D. et al. Simultaneous phase and amplitude extraction from a single defocused image of a homogeneous object. J Microsc 206(Pt 1), 33-40 (2002).

52. Schindelin, J. et al. Fiji: an open-source platform for biological-image analysis. Nat Methods 9(7), 676-82 (2012).

53. Roschger, P. et al. Bone mineralization density distribution in health and disease. Bone 42(3), 456-66 (2008).

54. Hesse, B. et al. Alterations of mass density and 3D osteocyte lacunar properties in bisphosphonate-related osteonecrotic human jaw bone, a synchrotron microCT study. PLoS One 9(2), e88481 (2014).

55. Giuliani, A. et al. Bisphosphonate-related osteonecrosis of the human jaw: A combined 3D assessment of bone descriptors by histology and synchrotron radiation-based microtomography. Oral Oncol 82, 200-202 (2018).

56. Weitkamp, T. et al. ANKAphase: software for single-distance phase retrieval from inline X-ray phase-contrast radiographs. J Synchrotron Radiat 18(Pt 4), 617-29. (2011).

57. Walker, C. L. \& Stewart, E. A. Uterine fibroids: the elephant in the room. Science 308(5728), 1589-1592 (2005).

58. Leppert, P. C., Catherino, W. H. \& Segars, J. H. A new hypothesis about the origin of uterine fibroids based on gene expression profiling with microarrays. Am J Obstet Gynecol 195(2), 415-420 (2006).

59. Stewart, E. A. et al. Relative overexpression of collagen type I and collagen type III messenger ribonucleic acids by uterine leiomyomas during the proliferative phase of the menstrual cycle. Journal of Clinical Endocrinology \& Metabolism 79(3), 900-906 (1994).

60. Norian, J. M. et al. Transforming growth factor beta3 regulates the versican variants in the extracellular matrix-rich uterine leiomyomas. Reproductive Sciences 16(12), 1153-1164 (2009).

61. Islam, M. S. et al. Role of activin-A and myostatin and their signaling pathway in human myometrial and leiomyoma cell function. Journal of Clinical Endocrinology \& Metabolism 99(5), E775-E785 (2014).

62. Leppert, P. C. et al. Comparative ultrastructure of collagen fibrils in uterine leiomyomas and normal myometrium. Fertility and Sterility 82(Suppl 3), 1182-1187 (2004).

63. Yoshino, O. et al. Decreased pregnancy rate is linked to abnormal uterine peristalsis caused by intramural fibroids. Hum Reprod 25(10), 2475-9 (2010).

64. Yoshino, O. et al. Myomectomy decreases abnormal uterine peristalsis and increases pregnancy rate. J Minim Invasive Gynecol 19(1), 63-7 (2012).

65. Jayes, F. L. et al. Loss of stiffness in collagen-rich uterine fibroids after digestion with purified collagenase Clostridium histolyticum. Am J Obstet Gynecol 215(5), 596 el-596 e8 (2016).

66. da Silva, A. P. et al. Histopathological and Digital Morphometrical Evaluation of Uterine Leiomyoma in Brazilian Women. Obstet Gynecol Int 2016, 2968410 (2016).

67. Flake, G. P. et al. The natural history of uterine leiomyomas: light and electron microscopic studies of fibroid phases, interstitial ischemia, inanosis, and reclamation. Obstet Gynecol Int 2013, 528376 (2013).

68. Jayes, F. L. et al. Evidence of biomechanical and collagen heterogeneity in uterine fibroids. PLoS One 14(4), e0215646 (2019).

69. Mommersteeg, T. J. et al. Nonuniform distribution of collagen density in human knee ligaments. J Orthop Res 12(2), 238-45 (1994).

70. Brosens, J. J., Barker, F. G. \& de Souza, N. M. Myometrial zonal differentiation and uterine junctional zone hyperplasia in the nonpregnant uterus. Hum Reprod Update 4(5), 496-502 (1998).

71. Ciavattini, A. et al. Uterine Fibroids: Pathogenesis and Interactions with Endometrium and Endomyometrial Junction. Obstetrics and Gynecology International 2013, 11 (2013).

72. Brun, F. et al. SYRMEP Tomo Project: a graphical user interface for customizing CT reconstruction workflows. Adv Struct Chem Imaging 3(1), 4 (2017).

73. Schneider, C. A., Rasband, W. S. \& Eliceiri, K. W. NIH Image to ImageJ: 25 years of image analysis. Nat Methods 9(7), 671-5 (2012).

74. Parfitt, A. M. et al. Bone histomorphometry: standardization of nomenclature, symbols, and units. Report of the ASBMR Histomorphometry Nomenclature Committee. J Bone Miner Res 2(6), 595-610 (1987).

75. Doube, M. et al. BoneJ: Free and extensible bone image analysis in ImageJ. Bone 47(6), 1076-9 (2010). 


\title{
Acknowledgements
}

The authors acknowledge the ELETTRA Synchrotron Facility User Office for kindly providing beam time for the propagation-based phase-contrast microCT investigations.

\section{Author Contributions}

A. Giuliani and P. Ciarmela designed research; G. Delli Carpini, S.R. Giannubilo and A. Ciavattini selected biopsies and data on patients; S. Pacilè and G. Tromba contributed with synchrotron-based microCT experiments and related analytic tools; A. Giuliani, S. Greco, S. Pacilè, A. Zannotti and P. Ciarmela performed research and data analysis; A. Giuliani and P. Ciarmela wrote the paper; while S. Greco, S. Pacilè, A. Zannotti, G. Delli Carpini, G. Tromba, S.R. Giannubilo and A. Ciavattini revised it critically for important intellectual content; ALL gave the final approval of the version to be published.

\section{Additional Information}

Supplementary information accompanies this paper at https://doi.org/10.1038/s41598-019-47048-0.

Competing Interests: The authors declare no competing interests.

Publisher's note: Springer Nature remains neutral with regard to jurisdictional claims in published maps and institutional affiliations.

\begin{abstract}
(c) (i) Open Access This article is licensed under a Creative Commons Attribution 4.0 International License, which permits use, sharing, adaptation, distribution and reproduction in any medium or format, as long as you give appropriate credit to the original author(s) and the source, provide a link to the Creative Commons license, and indicate if changes were made. The images or other third party material in this article are included in the article's Creative Commons license, unless indicated otherwise in a credit line to the material. If material is not included in the article's Creative Commons license and your intended use is not permitted by statutory regulation or exceeds the permitted use, you will need to obtain permission directly from the copyright holder. To view a copy of this license, visit http://creativecommons.org/licenses/by/4.0/.
\end{abstract}

(C) The Author(s) 2019 